\title{
Design of double dynamic vibration absorbers for reduction of two DOF vibration system
}

\author{
Lovely Son*, Mulyadi Bur ${ }^{\mathrm{a}}$, Meifal Rusli ${ }^{\mathrm{b}}$ and Adriyan \\ Department of Mechanical Engineering, Faculty of Engineering, Andalas University, \\ Kampus Limau Manis 25163, Indonesia
}

(Received October 23, 2015, Revised December 11, 2015, Accepted December 15, 2015)

\begin{abstract}
This research is aimed to design and analyze the performance of double dynamic vibration absorber (DVA) using a pendulum and a spring-mass type absorber for reducing vibration of two-DOF vibration system. The conventional fixed-points method and genetics algorithm (GA) optimization procedure are utilized in designing the optimal parameter of DVA. The frequency and damping ratio are optimized to determine the optimal absorber parameters. The simulation results show that GA optimization procedure is more effective in designing the double DVA in comparison to the fixed-points method. The experimental study is conducted to verify the numerical result.
\end{abstract}

Keywords: vibration; absorber; mass-spring; pendulum; GA

\section{Introduction}

The dynamic vibration absorber (DVA) is a device which is added on a body or structure to reduce vibration. The concept of DVA was firstly proposed by Frahm in 1909. Early studies outlined the basic theory and analytical method to optimize undamped and damped single mass DVA for the entire frequency range was conducted by Ormondroyd and Den Hartog in 1956.

DVAs are widely used on structures and machinery to passively reduce vibration. One application of DVA is for reducing vibration of tower suspension bridge (Casciati and Giuliano 2009). Other applications which DVAs were also used including controlling multi storey building vibrations (Seto et al. 2011), seismic protection of high towers (Giuseppe et al. 2008), reducing chatter during boring operation ( $\mathrm{Hu}$ et al. 2013) and suppressing image transfer belt system vibration (Yu et al. 2013). Nigdeli and Bekdas (2013) evaluate the application of TMD for preventing Brittle Fracture of RC Building.

The DVA performance will work effectively when the optimal DVA parameters are selected. For single degree of freedom (SDOF) vibration system, the optimal DVA parameters can be calculated directly using dynamic properties of SDOF main system. In this situation, the optimal natural frequency and damping ratio of the DVA are calculated as functions of DVA-structure mass

*Corresponding author, Ph.D., E-mail: lovelyson@ft.unand.ac.id

aProfessor, E-mail: mulyadibur@ft.unand.ac.id

${ }^{\text {b} P h . D ., ~ E-m a i l: ~ m e i f a l @ f t . u n a n d . a c . i d ~}$ 


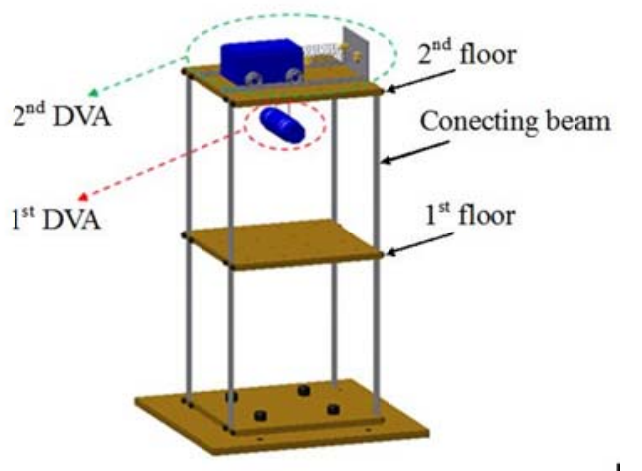

Fig. 1 Two-DOF building model using double DVA

ratio (Wu et al. 2009, Ghosh and Basu 2007).

Application of the active tuned vibration absorber (ATVA) for multi modes vibration problems has been investigated (Gong et al. 2012). Hashem and Hessamoddin (2014) propose an active tuned mass damper (ATMD) for controlling the seismic response of a multistory building.

Even thought the active tuned mass damper can reduce vibration over a broad frequency range and perform better than passive DVA, however for a large structure such as buildings and bridge, its needs the large actuator to realize the tuned parameters of the absorber. Recently, multiple TLCD have been used to suppress the vibration level of multi degree of freedom (MDOF) vibration system (Zhul et al. 2015). In the conventional design of multiple DVA for MDOF vibration system, the modal analysis approach usually used (Seto et al. 2011). By this method, the DVA for each vibration mode of the main system are separately designed using several decoupled SDOF systems which obtained by modal coordinate transformation method. The optimal DVAs frequency and corresponding damping ratio are then calculated using fixed-points method based on this decoupled SDOF system. However, in the real application, the characteristic of the main system usually change by shifting the natural frequency of the system due to the DVA addition. In this condition, the absorber design using decoupled SDOF main system will give non-optimal result.

Nowadays, some methods have been proposed by several researchers for designing the optimal DVA parameters (Ahn and Nguyen 2011, Xiang and Nishitani 2013, Yang et al. 2014, Bekdaş and Nigdeli 2011). Tributsch and Adam (2012) conduct optimal tuning of TMD parameters to obtain an accurate assessment of the TMD performance. Recently, optimum design of TMD for reducing the vibration response of adjacent structures has been developed (Nigdeli and Bekdas 2014).

In this research, a double-DVA system is applied to reduce the vibration of two-DOF building model. The first DVA consist of a SDOF pendulum system is used for reducing the first vibration mode and the second DVA consist of SDOF spring-mass system for reducing the second vibration mode of the main system. The schematic diagram of two-DOF building model using double DVA is depicted in Fig. 1.

Firstly, the absorber parameters are designed using conventional fixed-points method. The effect of the first and the second DVA masses on the performance of double DVA in reducing the main system vibration level is analyzed. In the second step, the optimum parameters of double DVA are calculated using genetics algorithm (GA) optimization procedure. The cost function for optimization is derived from frequency response function (FRF) of the vibration system. The 
effectiveness of DVA designed by GA will be compared to the conventional DVA design using fixed-points method. Finally, the experimental study is conducted to verify the simulation result.

\section{System modelling}

\subsection{Governing equation of the system}

Two-DOF spring-mass system is used to simplify the building model as shown in Fig. 1. Two dynamic absorbers consist of a pendulum and a spring-mass system are attached to the second mass of the main system as depicted in Fig. 2. It is assumed that the relative axial displacements of connecting beam shown in Fig. 1 are much smaller than its lateral displacement and the rotating motion of the floors are neglected. Therefore, the spring stiffness in horizontal motion can be calculated using theory of beam element for fixed-fixed boundary condition. This stiffness is then easily found as

$$
k_{b}=\frac{12 E_{b} I_{b}}{\left(\ell_{b}\right)^{3}}
$$

where $E_{b}, I_{b}$ and $\ell_{b}$ are elastic modulus, inertia moment and length of beam, respectively. Because of each floor are connected by four beam elements, the equivalent stiffness can be calculated by

$$
k_{e}=4 \times k_{b}=4 \times \frac{12 E_{b} I_{b}}{\left(\ell_{b}\right)^{3}}
$$

The governing equation of Two-DOF building using double dynamic absorber as shown in Fig. 2 can be written as

$$
\begin{gathered}
{\left[\begin{array}{cccc}
M_{f 1} & 0 & 0 & 0 \\
0 & M_{f 2}+m_{d 1} & 0 & m_{d 1} R \\
0 & 0 & m_{d 2} & 0 \\
0 & m_{d 1} R & 0 & m_{d 1} R^{2}
\end{array}\right]\left\{\begin{array}{c}
\ddot{x}_{1} \\
\ddot{x}_{2} \\
\ddot{x}_{d} \\
\ddot{\theta}
\end{array}\right\}+\left[\begin{array}{cccc}
0 & 0 & 0 & 0 \\
0 & c_{d 2} & -c_{d 2} & 0 \\
0 & -c_{d 2} & c_{d 2} & 0 \\
0 & 0 & 0 & c_{d 1}
\end{array}\right]\left\{\begin{array}{c}
\dot{x}_{1} \\
\dot{x}_{2} \\
\dot{x}_{d} \\
\dot{\theta}
\end{array}\right\}} \\
+\left[\begin{array}{cccc}
2 k_{e} & -k_{e} & 0 & 0 \\
-k_{e} & k_{e}+k_{d 2} & -k_{d 2} & 0 \\
0 & -k_{d 2} & k_{d 2} & 0 \\
0 & 0 & 0 & m_{d 1} g R
\end{array}\right]\left\{\begin{array}{c}
x_{1} \\
x_{2} \\
x_{d} \\
\theta
\end{array}\right\}=\left\{\begin{array}{c}
k_{e} \\
0 \\
0 \\
0
\end{array}\right\} X_{0} \sin \omega t
\end{gathered}
$$

or

$$
[\mathbf{M}]\{\ddot{\mathbf{x}}\}+[\mathbf{C}]\{\dot{\mathbf{x}}\}+[\mathbf{K}]\{\mathbf{x}\}=\{\mathbf{f}\}
$$

where $R$ is the pendulum length. For simplification purpose, the damping components of the main system are set to be zero. This assumption is realistic for structural damping of the main system used in this case study. However, the damping components of the absorber $c_{d 1}$ and $c_{d 2}$ are added into the governing equation of the system as depicted in Eq. (3). By using the modal analysis 


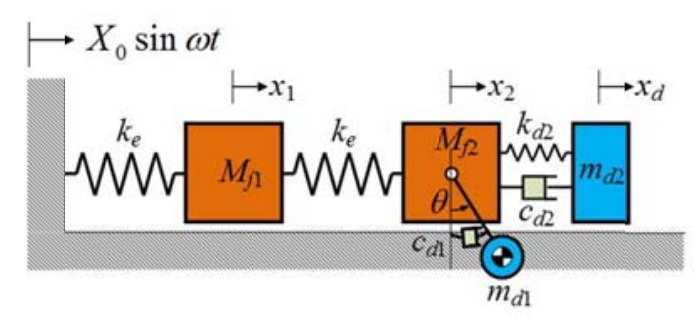

Fig. 2 Simple model of Two-DOF building using double DVA

technique, the governing equation in Eq. (4) can be written in modal coordinate as follows

$$
\left[\mathbf{m}_{\mathbf{r}}\right]\{\ddot{\mathbf{q}}\}+\left[\mathbf{c}_{\mathbf{r}}\right]\{\dot{\mathbf{q}}\}+\left[\mathbf{k}_{\mathbf{r}}\right]\{\mathbf{q}\}=\{\mathbf{p}\},\{\mathbf{q}\}=[\mathbf{\Phi}]^{-1}\{\mathbf{x}\} \text { and }\{\mathbf{p}\}=[\mathbf{\Phi}]\{\mathbf{f}\}
$$

or

$$
m_{r} \ddot{q}_{r}+c_{r} \dot{q}_{r}+k_{r} q_{r}=p_{r}, r=1,2, \cdots, N
$$

where $\boldsymbol{\Phi}, \mathbf{m}_{\mathbf{r}}, \mathbf{c}_{\mathbf{r}}$ and $\mathbf{k}_{\mathbf{r}}$ are the eigenvector, modal mass, modal damping and modal stiffness of the system, respectively. Meanwhile, $N$ is the degree of freedom of the structures with absorbers. The frequency response function which indicates the ratio between the system response and its excitation signal can be calculated as follows.

$$
\alpha_{j k}(\omega)=\sum_{r=1}^{N} \frac{\left(\Phi_{j r}\right)\left(\Phi_{k r}\right)}{\left(k_{r}-\omega^{2} m_{r}\right)+i\left(\omega c_{r}\right)}
$$

\subsection{Estimation of single degree of freedom model}

Lumped mass model of MDOF vibration system can be simplified into one SDOF system for each vibration mode at single point coordinate (Seto and Ookuma 1987). Base on this theory, the equivalent mass for single degree of freedom model for $i$-th vibration mode at the $j$-th coordinate point can be estimated by.

$$
M_{j}^{i}=\left\{\begin{array}{c}
x_{1} / x_{j} \\
\vdots \\
x_{j} / x_{j} \\
x_{N} / x_{j}
\end{array}\right\}_{i}^{T}\left[\begin{array}{llcc}
m_{1} & & & \\
& \ddots & 0 & \\
& 0 & m_{j} & \\
& & & m_{N}
\end{array}\right]\left\{\begin{array}{c}
x_{1} / x_{j} \\
\vdots \\
x_{j} / x_{j} \\
x_{N} / x_{j}
\end{array}\right\}_{i}
$$

The corresponding stiffness for each single degree of freedom of equivalent model at the $j$-th coordinate point and $i$ vibration mode is simply calculated from $K_{j}^{i}=M_{j}^{i} \omega_{i}^{2}$. Fig. 3 shows the transformation of Two-DOF vibration system into two equivalent SDOF systems at coordinate point $x_{2}$. The equivalent mass and stiffness for each vibration mode at this coordinate point are calculated as follows

$$
M_{2}^{1}=\left\{\begin{array}{l}
x_{1} / x_{2} \\
x_{2} / x_{2}
\end{array}\right\}_{1}^{T}\left[\begin{array}{cc}
m_{1} & 0 \\
0 & m_{2}
\end{array}\right]\left\{\begin{array}{l}
x_{1} / x_{2} \\
x_{2} / x_{2}
\end{array}\right\}_{1} \text { and } K_{2}^{1}=M_{2}^{1} \omega_{1}^{2}
$$




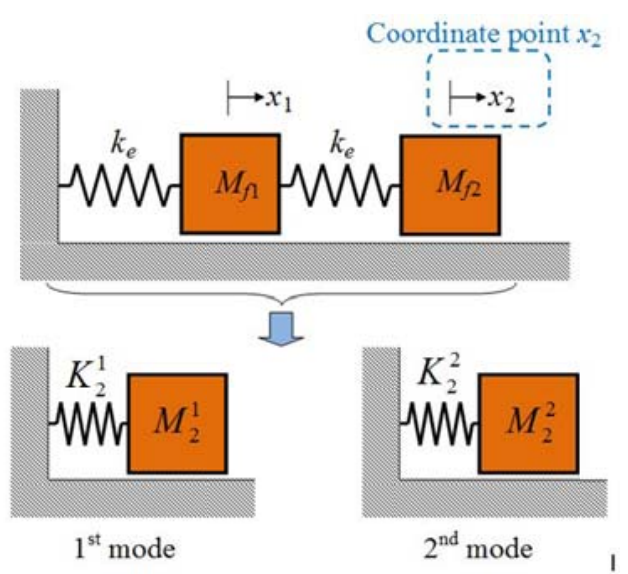

Fig. 3 Equivalent SDOF systems at coordinate point $x_{2}$

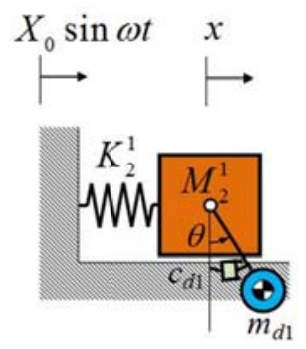

(a)

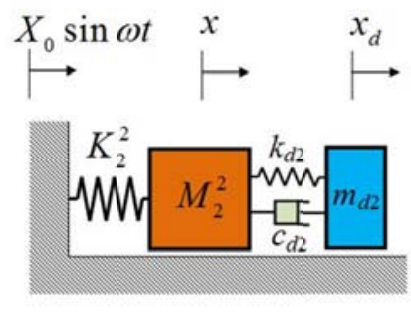

(b)

Fig. 4 Two SDOF equivalent systems with absorbers

$$
M_{2}^{2}=\left\{\begin{array}{l}
x_{1} / x_{2} \\
x_{2} / x_{2}
\end{array}\right\}_{2}^{T}\left[\begin{array}{cc}
m_{1} & 0 \\
0 & m_{2}
\end{array}\right]\left\{\begin{array}{l}
x_{1} / x_{2} \\
x_{2} / x_{2}
\end{array}\right\}_{2} \text { and } K_{2}^{2}=M_{2}^{2} \omega_{2}^{2}
$$

\section{Dynamic vibration absorber design}

\subsection{Two fixed-points method}

In designing DVA for two-DOF vibration system as shown in Fig. 2 using two fixed-points method, the absorbers designed separately for each vibration mode of the main system. Based on the system's mode shapes, the displacement response at the second mass are large enough for each vibration mode. For this reason, both absorbers are positioned at the second coordinate point $\left(x_{2}\right)$ as shown in Fig. 2. Absorber parameters are calculated separately using the equivalent SDOF system for each vibration modes at the second coordinate point. In this case, the pendulum absorber is used for reducing the vibration of the first vibration mode and the spring-mass absorber is applied for suppressing the vibration of the second vibration mode. The absorber parameters for each SDOF equivalent system as shown in Fig. 4 are then calculated using two fixed-points method. 


\subsubsection{Equivalent system with pendulum type absorber}

Fig. 4(a) shows the SDOF equivalent model of main system for the first vibration mode at $x_{2}$ coordinate point with pendulum type absorber. The stiffness and mass of this equivalent model are obtained using Eq. (8). Governing equation of the system are written in matrix form as

$$
\left[\begin{array}{cc}
M_{2}^{1}+m_{d 1} & m_{d 1} R \\
m_{d 1} R & m_{d 1} R^{2}
\end{array}\right]\left\{\begin{array}{c}
\ddot{x} \\
\ddot{\theta}
\end{array}\right\}+\left[\begin{array}{cc}
0 & 0 \\
0 & c_{d 1}
\end{array}\right]\left\{\begin{array}{c}
\dot{x} \\
\dot{\theta}
\end{array}\right\}+\left[\begin{array}{cc}
K_{2}^{1} & 0 \\
0 & m_{d 1} g R
\end{array}\right]\left\{\begin{array}{c}
x \\
\theta
\end{array}\right\}=\left\{\begin{array}{c}
K_{2}^{1} \\
0
\end{array}\right\} X_{0} \sin \omega t
$$

The normalized amplitude of the main system is calculated by

$$
\left|\frac{X}{X_{0}}\right|=\sqrt{\frac{\left(2 \zeta_{1} h_{1}\right)^{2}+\left(f_{1}^{2}-h_{1}^{2}\right)^{2}}{\left(2 \zeta_{1} h_{1}\right)^{2}\left[\left(1+\mu_{1}\right) h_{1}^{2}-1\right]^{2}+\left[h_{1}^{4}-\left(1+\mu_{1}\right) f_{1}^{2} h_{1}^{2}-\left(h_{1}^{2}-f_{1}^{2}\right)\right]^{2}}}
$$

where

$$
f_{1}=\frac{\omega_{d 1}}{\Omega_{1}} ; \quad \omega_{d 1}=\sqrt{\frac{g}{R}} ; \quad \Omega_{1}=\sqrt{\frac{K_{2}^{1}}{M_{2}^{1}}} ; \quad \zeta_{1}=\frac{c_{d 1}}{2 m_{d 1} \omega_{d 1} R^{2}} ; \quad \mu_{1}=\frac{m_{d 1}}{M_{2}^{1}} \quad \text { and } \quad h_{1}=\frac{\omega}{\Omega_{1}}
$$

\subsubsection{Equivalent system with mass-spring type aAbsorber}

The SDOF equivalent model of main system for the second vibration mode at the $x_{2}$ coordinate point with mass-spring type absorber is depicted in Fig. 4(b). The stiffness and mass parameters for this equivalent model are calculated using Eq. (9). Equations of motion of the system can be expressed in

$$
\left[\begin{array}{cc}
M_{2}^{2} & 0 \\
0 & m_{d 2}
\end{array}\right]\left\{\begin{array}{c}
\ddot{x} \\
\ddot{x}_{d}
\end{array}\right\}+\left[\begin{array}{cc}
c_{d 2} & -c_{d 2} \\
-c_{d 2} & c_{d 2}
\end{array}\right]\left\{\begin{array}{c}
\dot{x} \\
\dot{x}_{d}
\end{array}\right\}+\left[\begin{array}{cc}
K_{2}^{2}+k_{d 2} & -k_{d 2} \\
-k_{d 2} & k_{d 2}
\end{array}\right]\left\{\begin{array}{c}
x \\
x_{d}
\end{array}\right\}=\left\{\begin{array}{c}
K_{2}^{2} \\
0
\end{array}\right\} X_{0} \sin \omega t
$$

The main system normalized amplitude is written by

$$
\left|\frac{X}{X_{0}}\right|=\sqrt{\frac{\left(2 \zeta_{2} h_{2}\right)^{2}+\left(f_{2}^{2}-h_{2}^{2}\right)^{2}}{\left(2 \zeta_{2} h_{2}\right)^{2}\left[\left(1+\mu_{2}\right) h_{2}^{2}-1\right]^{2}+\left[h_{2}^{4}-\left(1+\mu_{2}\right) f_{2}^{2} h_{2}^{2}-\left(h_{2}^{2}-f_{2}^{2}\right)\right]^{2}}}
$$

where

$$
f_{2}=\frac{\omega_{d 2}}{\Omega_{2}} ; \quad \omega_{d 2}=\sqrt{\frac{k_{d 2}}{m_{d 2}}} ; \quad \Omega_{2}=\sqrt{\frac{K_{2}^{2}}{M_{2}^{2}}} ; \quad \zeta_{2}=\frac{c_{d 2}}{2 m_{d 2} \omega_{d 2}} ; \mu_{2}=\frac{m_{d 2}}{M_{2}^{2}} \text { and } h_{2}=\frac{\omega}{\Omega_{2}}
$$

\subsubsection{Optimum tuning frequency and damping ratio}

The optimum tuning frequency of absorber using two fixed-points method has been derived by Den-Hartog (1956). The optimum tuning frequency for the pendulum and mass-spring type absorber are expressed as follows

$$
f_{o p t 1}=\frac{1}{1+\mu_{1}} ; f_{o p t 2}=\frac{1}{1+\mu_{2}}
$$


The optimum damping ratios are given by

$$
\zeta_{\text {opt } 1}^{2}=\frac{3 \mu_{1}}{8\left(1+\mu_{1}\right)} ; \quad \zeta_{o p t 2}^{2}=\frac{3 \mu_{2}}{8\left(1+\mu_{2}\right)}
$$

\subsection{GA optimization procedure}

The genetic algorithm (GA) is an optimization and search technique based on the principles of genetics and natural selection (Haupt and Haupt 2004). This method selects one population composed of many individuals utilizing specified criteria, which minimize the cost function. The method was developed by John Holland and popularized by one of his students, David Goldberg.

In the case of dynamic absorber design, generally two optimization criteria can be used for obtaining the optimal absorber parameters. In the first criterion, the objective is to minimize the maximum amplitude ratio of the response of the primary system to excitation force or motion. In another criterion, the objective is for reducing the total vibration energy of the system in the overall frequencies.

In this research, the first optimization criterion is used for calculating the optimum parameters of dynamic absorber using GA procedure. To achieve this purpose, the objective function is formulated as the sum of the maximum amplitude ratio in the frequency region that is close to each natural frequency of the main system. For two-DOF building model with dynamic absorbers as shown in Fig. 1, the cost function is calculated by

$$
\text { Cost function }=\max \left(w F_{1}\right)+\max \left((1-w) F_{2}\right)
$$

Variable $w$ in Eq. (16) denotes the weighting number. Variables $F_{1}$ and $F_{2}$ are functions which describe the amplitude ratio between response and excitation signal. These two functions are evaluated for frequency range near to the first and the second natural frequency of main structure. These amplitude ratios can be obtained by calculating the absolute value of frequency response function as given by

$$
F=\left|\alpha_{j k}(\omega)\right|=\left|\sum_{r=1}^{N} \frac{\left(\Phi_{j r}\right)\left(\Phi_{k r}\right)}{\left(k_{r}-\omega^{2} m_{r}\right)+i\left(\omega c_{r}\right)}\right|
$$

Where

$$
\begin{gathered}
F_{1}=F \text { at } \omega_{L_{1}}<\omega<\omega_{R_{1}} \\
F_{2}=F \text { at } \omega_{L_{2}}<\omega<\omega_{R_{2}}
\end{gathered}
$$

Variables $\omega_{L 1}$ and $\omega_{R 1}$ denote the left and right side boundary of the first frequency function. The same rule is also applied to $\omega_{L 2}$ and $\omega_{R 2}$ for the second frequency function. The optimized variables for optimization are frequency ratios $f_{1}$ and $f_{2}$ and damping ratios $\zeta_{1}$ and $\zeta_{2}$. For two-DOF building model with double dynamic absorbers, variable $f_{1}$ and $f_{2}$ are calculated using Eq. (14). Meanwhile, variable $\zeta_{1}$ and $\zeta_{2}$ are calculated from Eq. (15).

Fig. 5 shows the GA algorithm procedure in calculating the optimal parameter of double dynamic vibration absorber. The algorithm begins with defining the cost function, variables and GA parameters such as population size, number of parameters, fraction of population kept and 


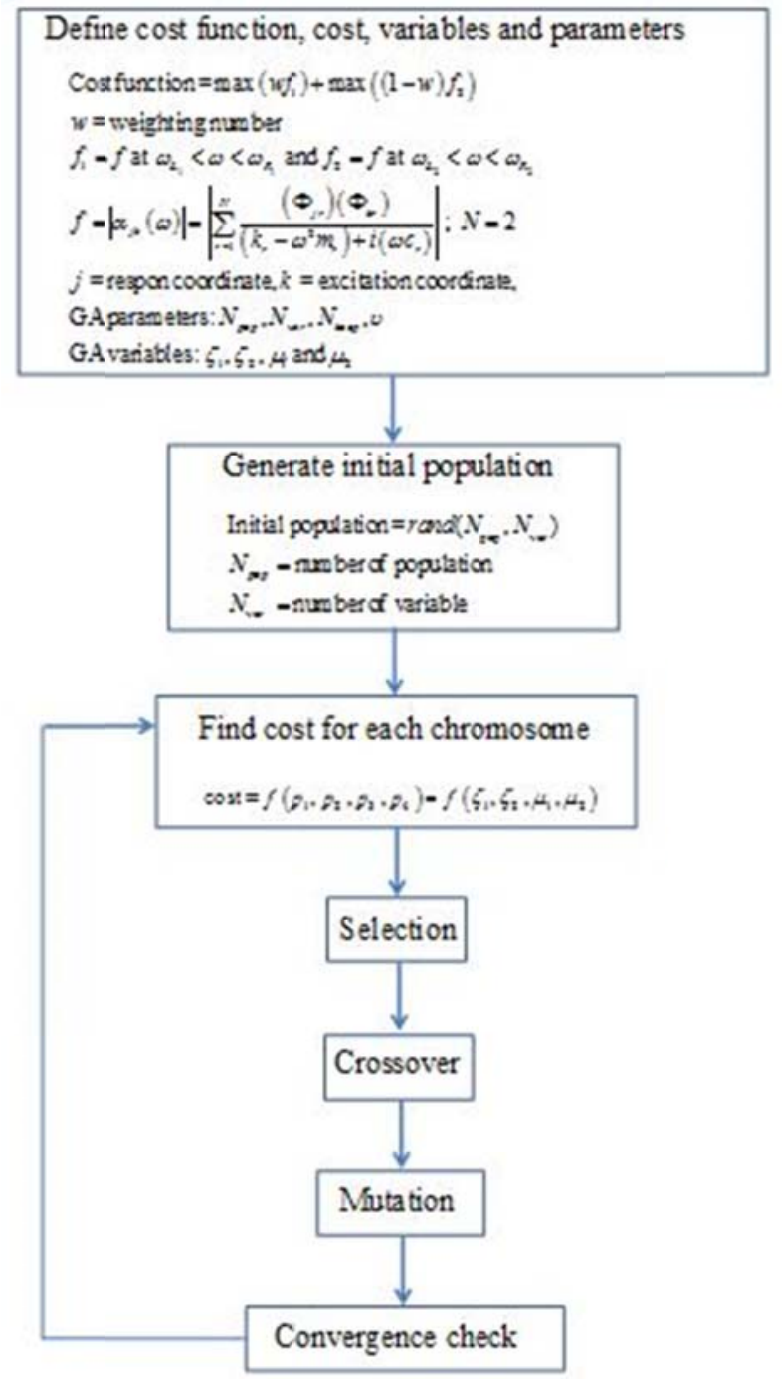

Fig. 5 GA procedure

mutation rate. In the second step, the initial population is generated. Next, the costs of each population members are calculated for selection process. The selected members in population at each generation are processed using GA operation i.e., crossover and mutation for obtaining the optimal value of the function. The GA optimization procedures are conducted iteratively until the process stopped by the convergence check.

\section{Numerical study}

In order to explore the effectiveness of pendulum and spring-mass type double DVA, its performance is evaluated using two design method. Firstly, the DVA parameters are designed by 


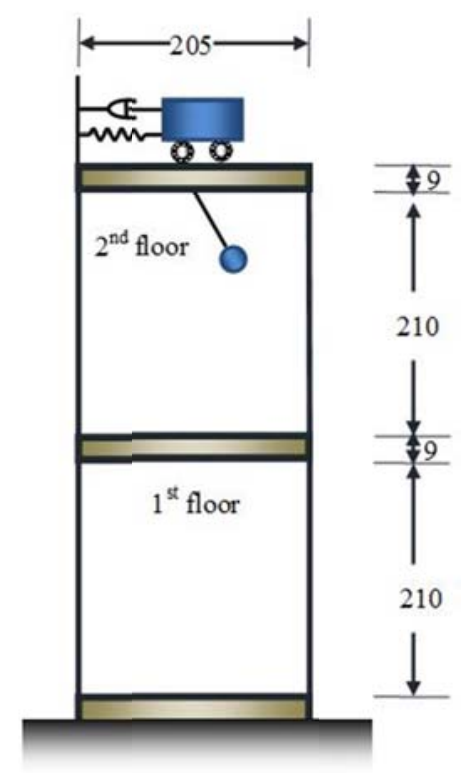

Fig. 6 Schematic diagram of building model

Table 1 Simulation parameters

\begin{tabular}{ccc}
\hline \hline No & Parameters & Value \\
\hline 1 & Mass of the $1^{\text {st }}$ floor $\left(M_{f 1}\right)$ & $3.035 \mathrm{~kg}$ \\
2 & Mass of the $2^{\text {nd }}$ floor $\left(M_{f 2}\right)$ & $3.010 \mathrm{~kg}$ \\
3 & Beam length $\left(\ell_{b}\right)$ & $210 \mathrm{~mm}$ \\
4 & Beam inertia moment $\left(I_{b}\right)$ & $1.667 \mathrm{~mm}^{4}$ \\
5 & Beam elastic modulus $\left(E_{b}\right)$ & $190 \mathrm{MPa}$ \\
6 & Beam cross sectional area $(A)$ & $20 \mathrm{~mm}^{2}$ \\
\hline
\end{tabular}

conventional two fixed-points method. Next, the optimal parameters of DVA are calculated using GA optimization procedure. The performance of DVA designed by these two methods is evaluated. The design purpose is to optimize the frequency and damping ratio of the absorber.

Fig. 6 shows the schematic diagram of building model with double dynamic vibration absorbers used in the simulation. The simulation parameters are shown in Table 1. The building model consists of two lumped masses made of steel plate that connected using four steel beams. The beam length is much longer than its width and thickness. Therefore, these beams can be assumed as four leaf spring with stiffness given by Eq. (1). The base of building is fixed to the ground. The ground is excited in horizontal direction.

Numerical simulation of building model is conducted in MATLAB programing language. The modal analysis technique is used to obtain the modal parameters and response of the structure. It is found that the structure natural frequencies of the $1^{\text {st }}$ and $2^{\text {nd }}$ modes are $2.24 \mathrm{~Hz}$ and $5.79 \mathrm{~Hz}$, respectively. The structural damping is assumed very small in comparison to the damping of the vibration absorber. These structural damping for the first and the second vibration mode are identified as 0.025 and 0.01 , respectively. 


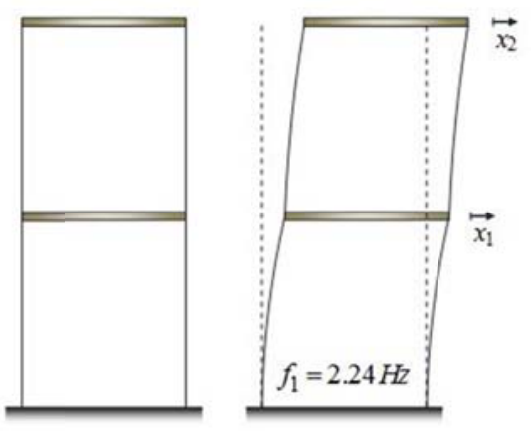

(a)

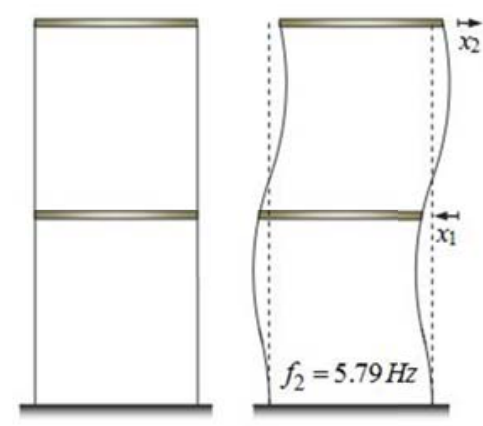

(b)

Fig. 7 Mode shape and natural frequency of two-DOF building model The first mode (b) The second mode

Table 2 GA optimization parameters

\begin{tabular}{ccc}
\hline \hline No & Parameters & Value \\
\hline 1 & Population size $\left(N_{p o p}\right)$ & 16 \\
2 & Mutation rate $(v)$ & 0.2 \\
3 & Fraction of population kept $(\sigma)$ & 0.5 \\
4 & Number of parameters $\left(N_{v a r}\right)$ & 4 \\
5 & Weighting number $(w)$ & 0.5 \\
\hline
\end{tabular}

\subsection{Simulation using harmonic excitation}

The first two vibration mode shapes and corresponding natural frequency are shown in Fig. 7. By utilizing these mode shapes, the equivalent mass for the first and the second mode at the second floor as calculated by Eq. (8) and Eq. (9) are given by

$$
M_{2}^{1}=4.17 \mathrm{~kg}, \quad M_{2}^{2}=10.80 \mathrm{~kg}
$$

The absorber mass is calculated using the structure equivalent mass and the absorber mass ratio. In the simulation, the absorber mass ratios are selected manually. For experimental verification in the next chapter, the absorber mass ratio are chosen as $\mu_{1}=0.12$ and $\mu_{2}=0.052$. For given mass ratio, the absorber mass are obtained as $m d_{1}=0.5 \mathrm{~kg}$ and $m d_{2}=0.56 \mathrm{~kg}$. Using these mass ratio, the optimal frequency and damping ratio for each absorber are calculated using Eqs. (14) and (15).

In GA optimization procedure, the optimal values of absorber frequency ratio and damping ratio are obtained by random search technique. The variable constraint for frequency ratio and damping ratio used in GA optimization simulation are $0.001 \leq f_{1} \leq 1,0.001 \leq f_{2} \leq 1,0 \leq \zeta_{1} \leq 0.02$ and $0 \leq \zeta_{2} \leq 0.02$. The GA parameters used in simulation are given in Table 2 . The frequency limit for $F_{1}$ and $F_{2}$ in Eqs. (18) and (19) are selected 0 to $5 \mathrm{~Hz}$ and 5 to $10 \mathrm{~Hz}$, respectively.

The optimal parameters of DVA such as pendulum length $(R)$ for the $1^{\text {st }}$ absorber and spring stiffness $\left(k_{d 2}\right)$ for the $2^{\text {nd }}$ absorber are obtained using the optimal value of frequency ratio. The absorber damping $c_{d 1}$ and $c_{d 2}$ are calculated using the optimal value of damping ratio. Table 3 show the optimal parameters of DVA obtained using conventional fixed-points and GA optimization method. 
Table 3 Optimal DVA parameters

\begin{tabular}{cccc}
\hline \hline & \multirow{2}{*}{ Parameters } & \multicolumn{2}{c}{ Optimal value } \\
\cline { 2 - 4 } & $f_{1}$ & Fixed-points method & GA \\
\hline \multirow{4}{*}{ DVA 1 } & $\zeta_{1}$ & 0.893 & 0.854 \\
& $R$ & 0.200 & 0.200 \\
& $c_{d 1}$ & $0.059 \mathrm{~m}$ & $0.065 \mathrm{~m}$ \\
& $f_{2}$ & $0.009 \mathrm{Ns}$ & $0.010 \mathrm{Ns}$ \\
\hline \multirow{4}{*}{ DVA2 } & $\zeta_{2}$ & 0.951 & 0.899 \\
& $k_{d 2}$ & 0.136 & 0.200 \\
& $c_{d 2}$ & $720.3 \mathrm{~N} / \mathrm{m}$ & $642.5 \mathrm{~N} / \mathrm{m}$ \\
& & $5.478 \mathrm{Ns} / \mathrm{m}$ & $7.591 \mathrm{Ns} / \mathrm{m}$ \\
\hline
\end{tabular}

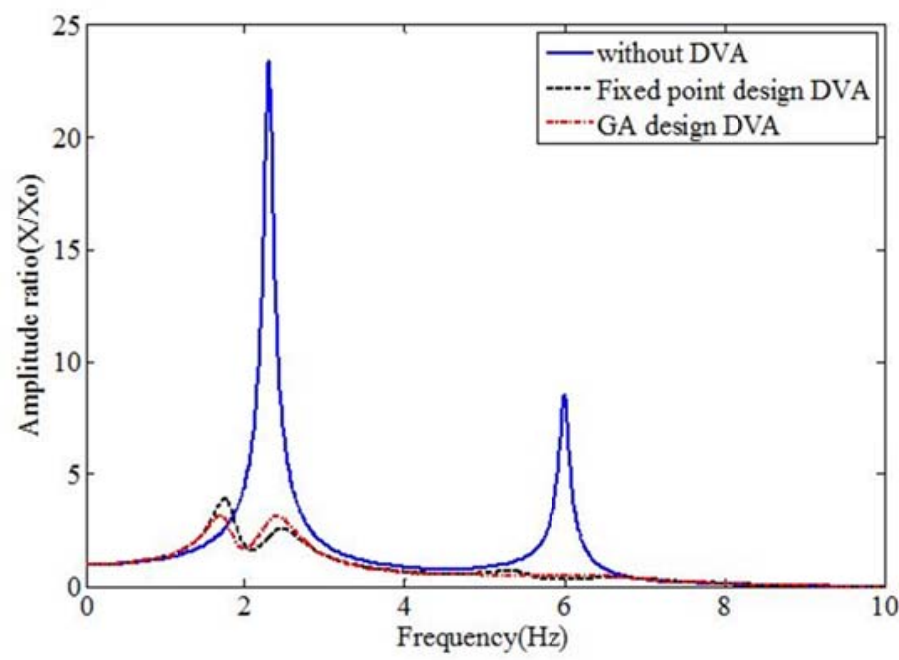

Fig. 8 Simulation result of amplitude ratio

Fig. 8 shows the amplitude ratio between displacement response at the second floor and displacement of the ground. The simulation result depicted in Fig. 8 shows that the fixed-points design of DVA reduces the amplitude ratio in region close the first and the second natural frequency of the structure. However, the difference peak level at the left and right side of first natural frequency $(2.24 \mathrm{~Hz})$ due to absorber addition shows that the conventional method produces not optimum DVA parameter. This condition also obtained for the second absorber which tuned to the second natural frequency of the structure. In the case of absorber design using GA procedure, the peak amplitude ratio at the left and the right side of the structure naturall frequency has the same level. These results show that absorber design using GA optimization procedure has superior performance in comparison to the fixed-points method.

The conventional design of double DVA using fixed-points method utilizes the eigenvector of Two-DOF structure for determining the equivalent masses at the absorber location. These equivalent masses are used for calculation of optimal absorber parameters such as length of pendulum $(R)$ and the stiffness of spring- mass system $\left(k_{2}\right)$. The mass contribution of one absorber 


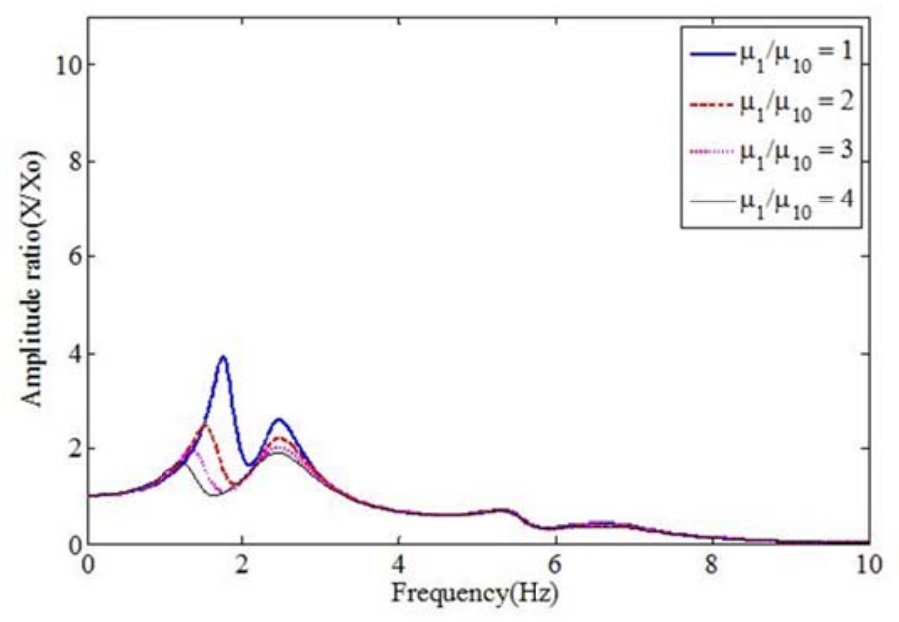

Fig. 9 Simulation of amplitude ratio with variation of $\mu_{1}$

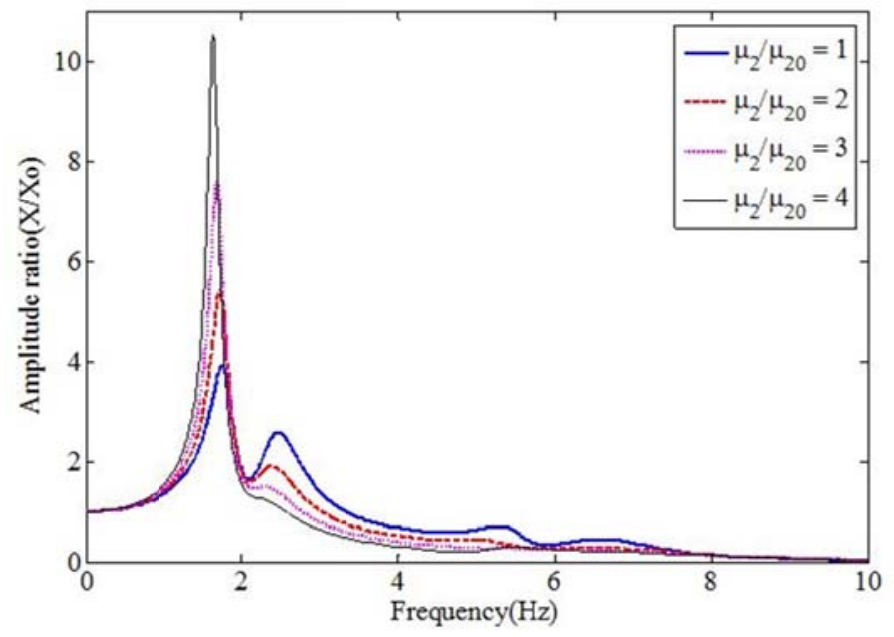

Fig. 10 Simulation of amplitude ratio with variation of $\mu_{2}$

will cause variation of the structure's eigenvector. Therefore, the variation of absorber mass will reduce the absorber performance. However, in the GA optimization procedure this problem is not found due to the random search process during GA calculation will drive the calculation result to the global optimum condition.

The effect of $1^{\text {st }}$ absorber mass variation to the amplitude ratio in the case of conventional fixed-points design is shown in Fig. 9. In the simulation, the mass ratio of the first absorber is varied from 1 to 4 times the reference value $\left(\mu_{10}=0.12\right)$. The reference value for mass ratio is selected the same as that used in experimental study in next chapter. It can be seen from Fig. 9 that the $1^{\text {st }}$ absorber mass variations significantly reduce the absorber performance.

The effect of the second absorber mass variation to the absorber performance for conventional DVA design is illustrated in Fig. 10. In this simulation, the $2^{\text {nd }}$ absorber mass ratio is varied from 1 to 4 reference value used in experimental study $\left(\mu_{20}=0.052\right)$. Almost similar condition obtained 


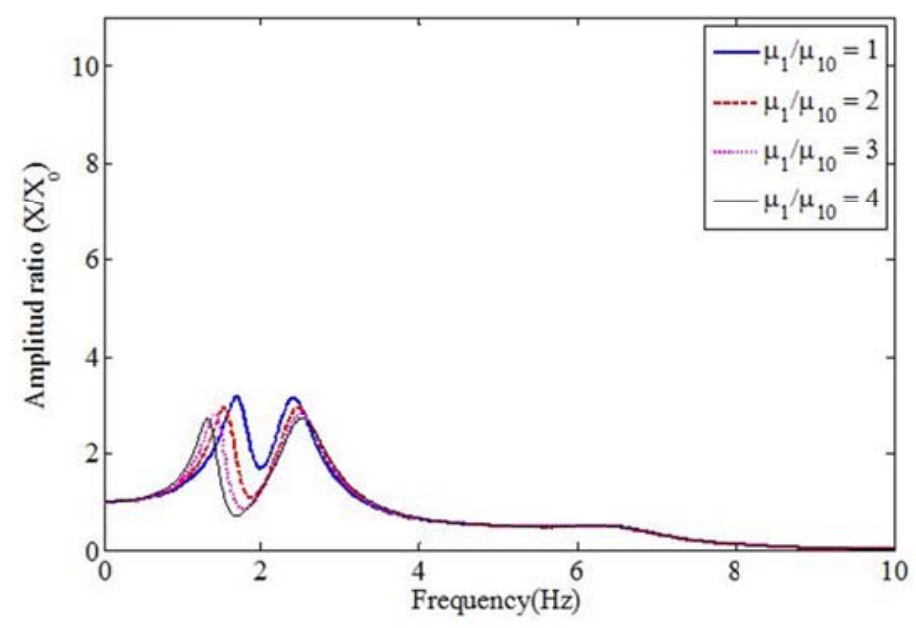

Fig. 11 GA simulation of amplitude ratio with variation of $\mu_{1}$

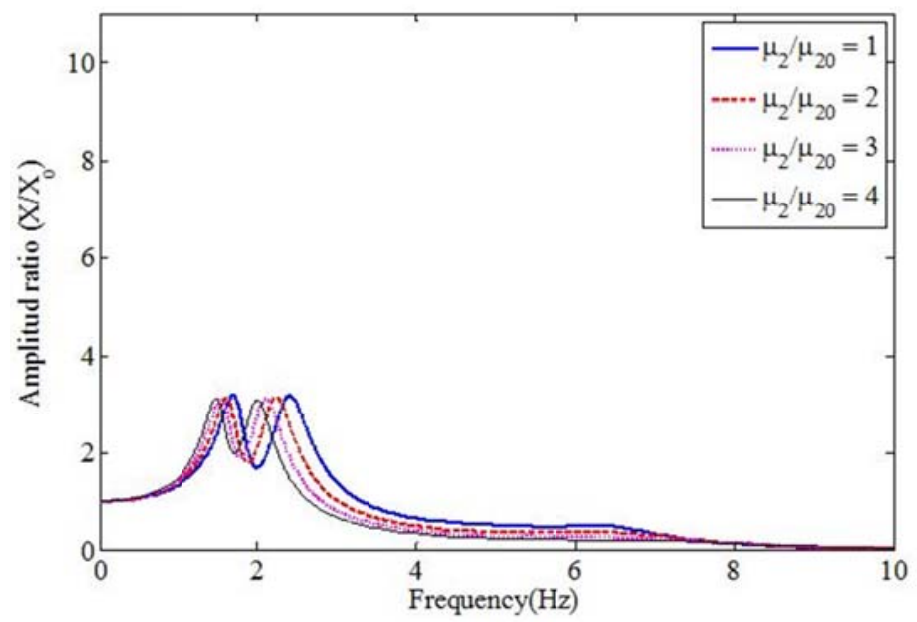

Fig. 12 GA simulation of amplitude ratio with variation of $\mu_{2}$

with the $1^{\text {st }}$ absorber variation is detected in the second absorber variation. Even though the variation of amplitude ratio close to $2^{\text {nd }}$ natural frequency is very small, but larger variation of amplitude ratio is found near to $1^{\text {st }}$ natural frequency.

The simulation results depicted in Fig. 9 and Fig. 10 show that variation of DVA mass ratio will reduce the performance of double dynamic absorber. Moreover, performance of the first DVA in reducing of the first resonance frequency response is very sensitive to variation of the absorber mass ratio. This is because the contribution of the first mode shape to the system response is larger than that of the second mode shape.

Fig. 11 shows the influence of the first absorber mass ratio to the DVA performance using GA design procedure. In the GA simulation, the weighting factor is selected as $w=0.5$. It can be shown from Fig. 11 that the peaks resonance will reduce when the first absorber mass ratio is increased. However, the optimal condition of the absorber does not significantly change because the 


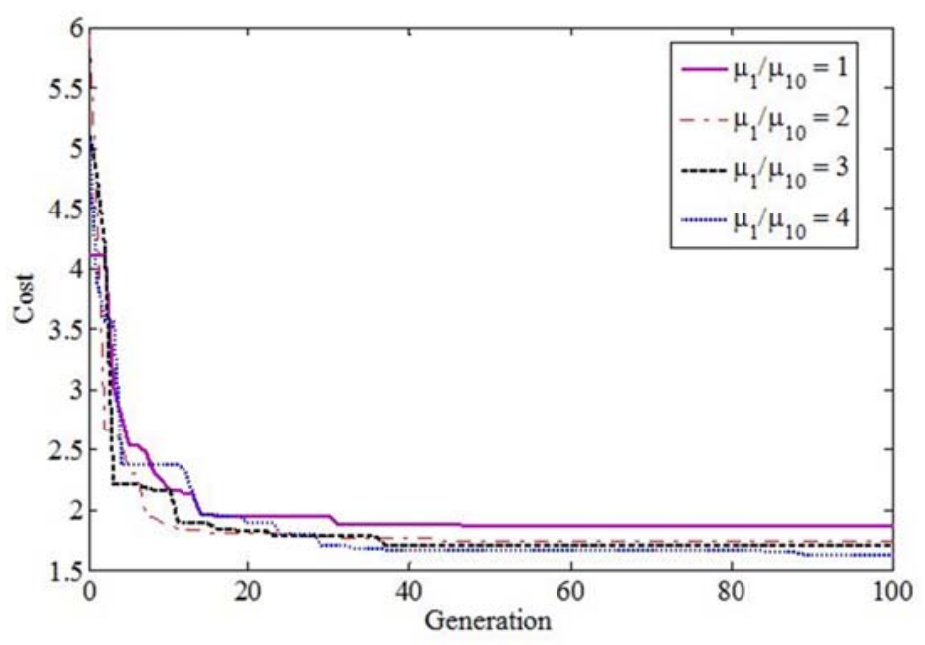

Fig. 13 History of cost function in each generation
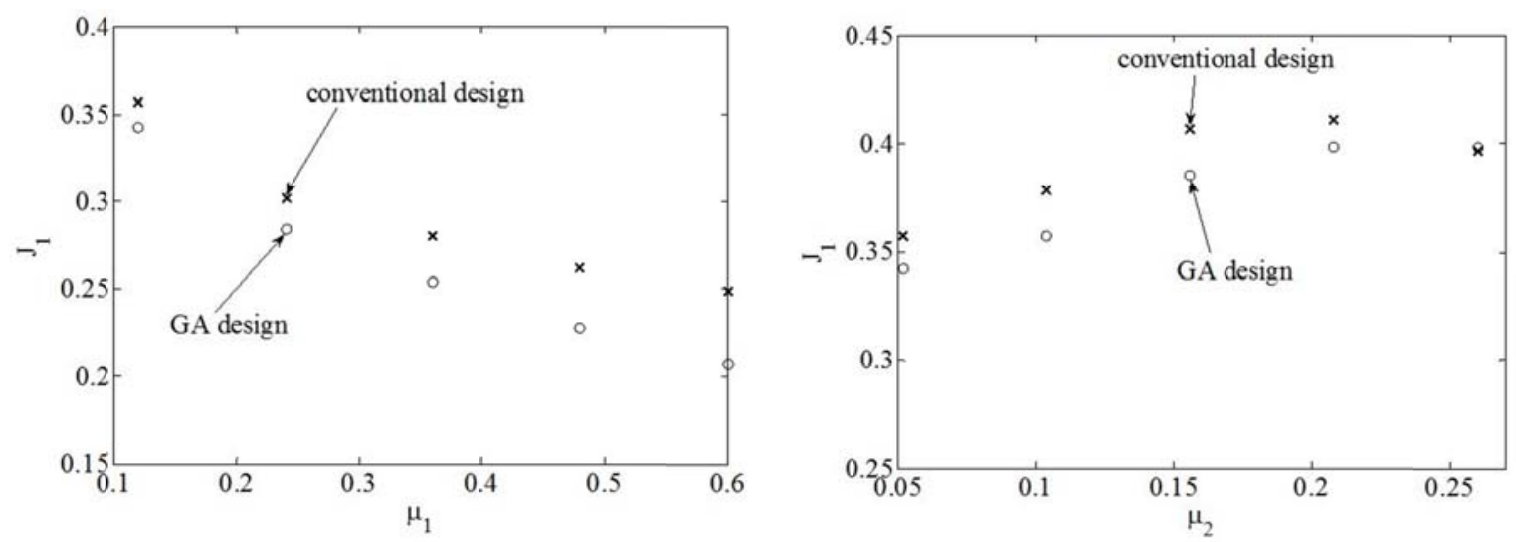

Fig. 14 Variation of $J_{1}$ vs mass ratio under El Centro earthquake

difference between two peaks resonance near to the first natural frequency is very small.

In the case of $2^{\text {nd }}$ absorber mass variation, the absorber design using GA procedure shows the better performance as shown in Fig. 12 in comparison to the conventional design using fixedpoints method (Fig. 10). As shown in Fig. 12, variation of the $2^{\text {nd }}$ absorber mass only shift two peak frequency response near to the $1^{\text {st }}$ natural frequency but the relative height of these two peaks resonance are not significantly change.

Fig. 13 shows the cost function history in each generation of GA procedure. The cost function is evaluated when the first DVA mass ratio varied from 1 to 4 . It is shown in Fig. 13 that the optimization procedure becomes stable after 40 generation. This result indicates that the GA optimization used in this paper is a stable optimization technique.

\subsection{Simulation using random excitation}

To evaluate the damper performance in the case of random excitation, the model of the building 

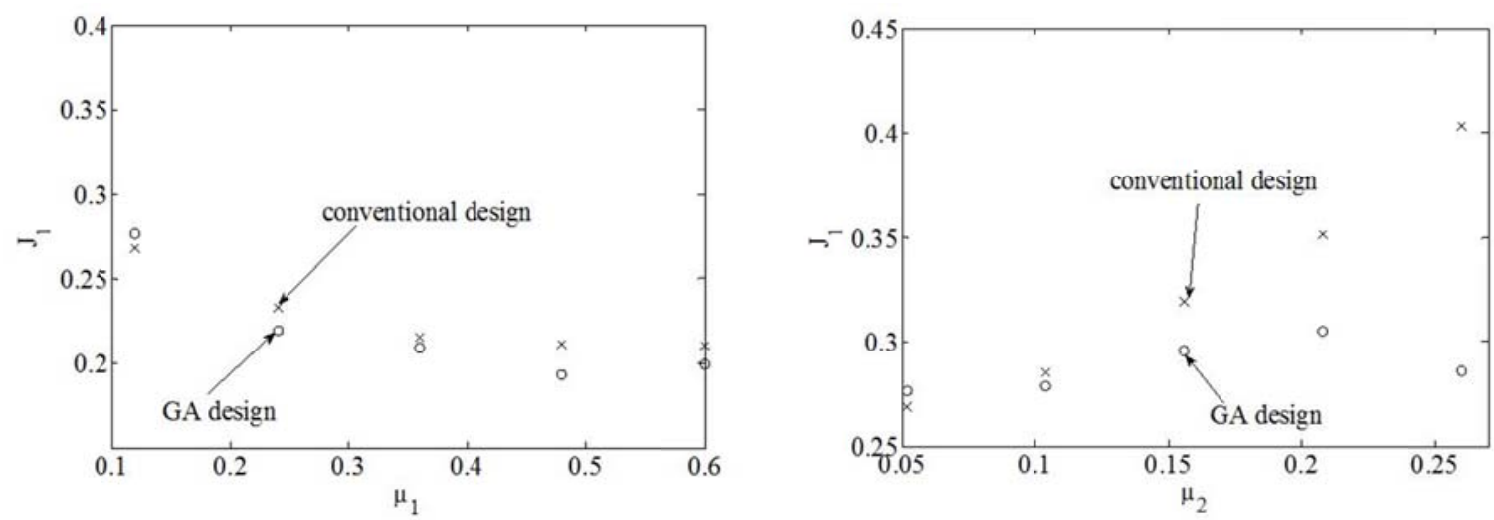

Fig. 15 Variation of $J_{1}$ vs mass ratio under Kobe earthquake

structure is subjected to the two earthquakes, El Centro earthquake (1940) and Kobe earthquake (1995). Comparison of double dynamic vibration absorber performance using conventional and GA design procedure are evaluated using evaluation criterion $J_{1}$ which is a measure of the normalized displacement of the second floor. Here, the displacement responses are normalized to those obtained without DVA.

Fig. 14 shows the performance index $\left(J_{1}\right)$ variation calculated under El Centro earthquake excitation. The performance indexes are calculated from displacement responses obtained using two design procedures for DVA i.e., conventional and GA design method. The left hand Fig. 14 shows the variation of $J_{1}$ vs $\mu_{1}$ and the right hand one depicts the relation between $J_{1}$ and $\mu_{2}$. The results shown in Fig. 14 indicate that the performance indexes obtained using GA design procedure are smaller than those obtained using conventional design procedure.

The performance index variation versus mass ratio calculated under Kobe earthquake excitation is shown in Fig. 15. It can be seen from Fig. 15 that the performance index of the structure responses using DVA designed by GA method has lower values in comparison to those obtained by conventional absorber design.

\section{Experiment}

The experimental model of two-DOF building is constructed for verification of the numerical result. The dimensions and other parameters of the experimental model are the same as those in the numerical study as shown in Table 1. The natural frequencies and mode shapes obtained from impact test of the experimental model are also the same as used in numerical study as depicted in Fig. 6. The DVA parameters such as mass of DVA1 $\left(m_{d 1}\right)$, mass of DVA2 $\left(m_{d 2}\right)$, length of pendulum $(R)$ and stiffness of DVA2 $\left(k_{2}\right)$ are selected equal to those used in simulation study. However, the damping factor of DVA1 $\left(\zeta_{1}\right)$ and DVA2 $\left(\zeta_{2}\right)$ are very difficult to be tuned in the experimental study. Therefore in the experiment, the default values of damping factor were used. These default which calculated by fitting the simulation and experimental response are $\zeta_{1}=0.07$ and $\zeta_{2}=0.07$.

The experimental data are collected using PULSE digital signal analyzer. The photograph of experimental set-up is shown in Fig. 14. Two dynamic vibration absorbers i.e., mass-spring and 


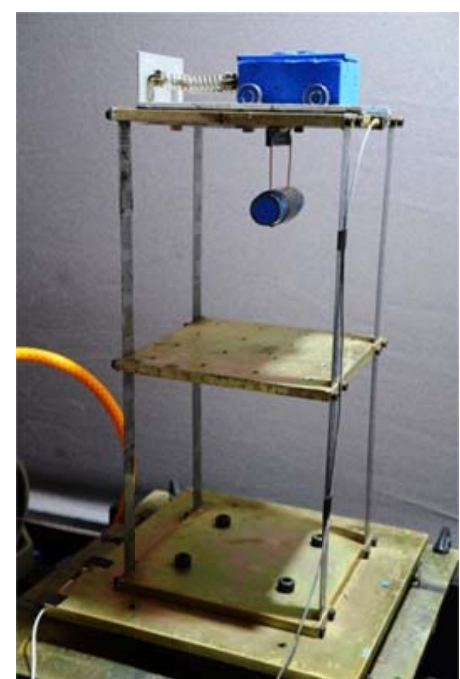

Fig. 16 Photograph of experimental set-up

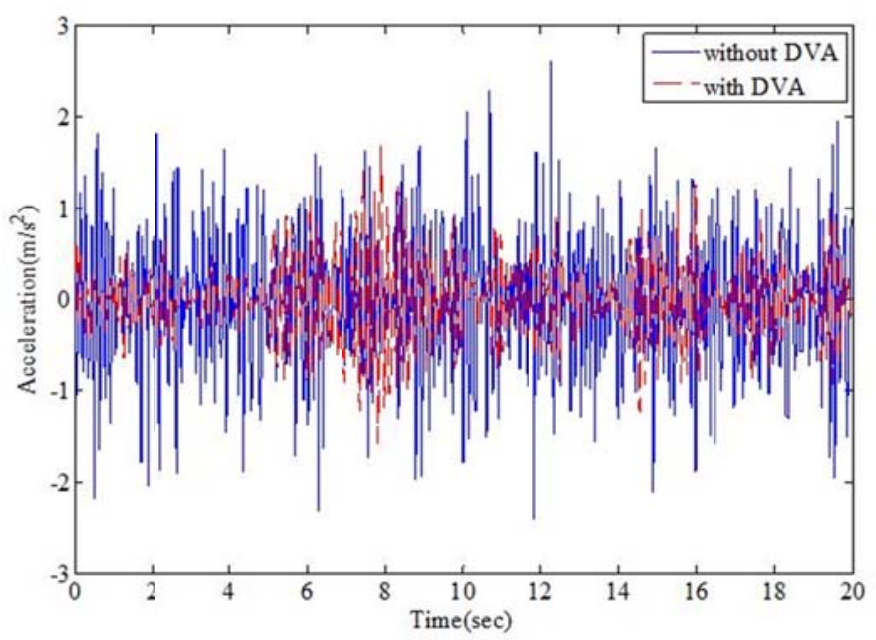

Fig. 17 Experimental result of $2^{\text {nd }}$ floor acceleration response

pendulum systems are positioned at the $2^{\text {nd }}$ floor of the building models. The absorbers damping are dominantly caused by friction phenomena. The first absorber damping is mainly due to friction between mass and floor while the second absorber damping come from pin connected pendulum. The first absorber mass made of wood block which is supported by two helical springs in horizontal direction. The pendulum system consists of a rod mass that hanged using two copper rods. These rods are pin connected to the second floor as shown in Fig. 16. The acceleration response of the second floor and the excitation signal at the base structure are measured using accelerometer. The random excitation signal was used to excite the base structure in experimental study. The acceleration data of the $2^{\text {nd }}$ floor and the base structure are processed offline in time and frequency domain using MATLAB software. The response signal is filtered by $10 \mathrm{~Hz}$ Low Pass Filter. A Hanning window is used to reduce the spectral leakage of sampled signal. This window is 


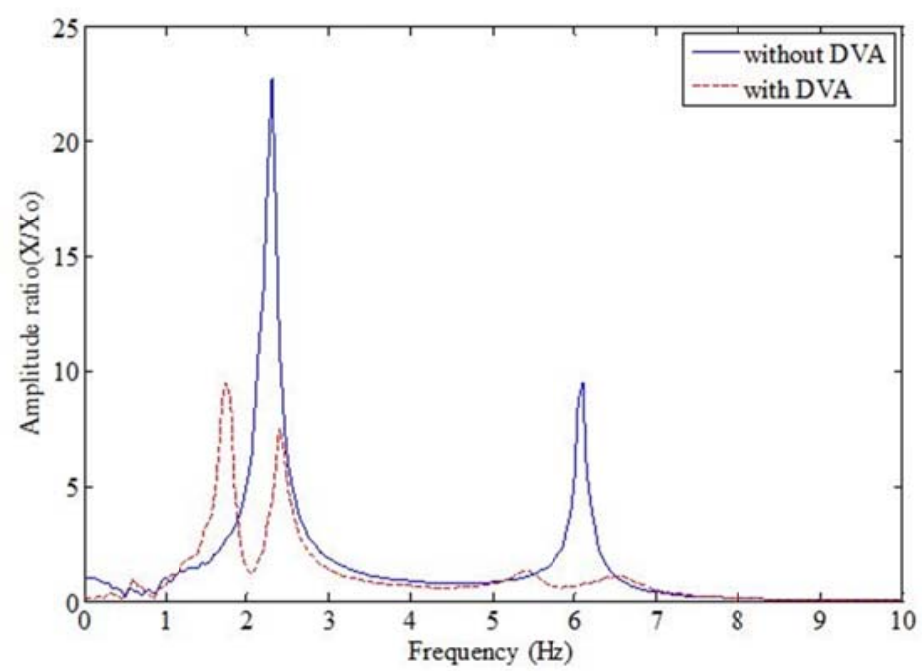

Fig. 18 Experimental result of amplitude ratio

aimed to improve the spectral characteristic of sampled signal.

Fig. 17 shows the acceleration response measured at the $2^{\text {nd }}$ floor. Continues line in Fig. 15 shows the response of the structure without DVA and dashed line indicates the structure response with DVA. The structure responses are significantly affected by the $1^{\text {st }}$ and the $2^{\text {nd }}$ modes component. The attenuation of response due to dynamic absorber is shown clearly.

The amplitude ratio between response of the structure at the $2^{\text {nd }}$ floor and excitation signal is calculated and displayed in frequency domain as shown in Fig. 18. It can be shown that the peak responses close to $1^{\text {st }}$ and $2^{\text {nd }}$ natural frequency are reduced significantly.

\section{Conclusions}

Vibration suppression of two-DOF vibration system using double dynamic vibration absorber has been purposed. The absorbers consist of a pendulum and a spring-mass vibration system which are used to reduce the response at the first and the second structure natural frequency, respectively. Two design approach using conventional fixed-points method and Genetic Algorithm (GA) optimization procedure are utilized in obtaining the optimum absorber parameters. It is find that the conventional design of absorber using fixed-points method very sensitive to the absorber mass variation. Meanwhile, the absorber design using GA procedure is robust to the parameters variation. This is due to the random search in GA procedure will drive the optimization process to the global optimum of absorber parameters.

\section{Acknowledgments}

Financial support by Hibah Bersaing research Fund from Directorate General of Higher Education of Indonesia (Grant No.Dipa-023.04.2.415061/2013) are gratefully acknowledged. 


\section{References}

Ahn, N.D. and Nguyen, N.X. (2011), "Extension of equivalent linearization method to design of TMD for linear damped systems", Struct. Control Hlth. Monit., 19(6), 565-573.

Bekdaş, G. and Nigdeli, S.M. (2011), "Estimating optimum parameters of tuned mass dampers using harmony search", Eng. Struct., 33(9), 2716-2723.

Casciati, F. and Giuliano, F.(2009), "Performance of Multi-TMD in the Towers of Suspension Bridges", $J$. Vib. Contr., 15(6), 821-847.

Den Hartog, J.P. (1956), Mechanical Vibrations: Fourth Edition, McGraw-Hill Book Company, New York, USA.

Ghosh, A. and Basu, B. (2007), "A closed-form optimal tuning criterion for TMD in damped structures", Struct. Control Hlth. Monit., 14, 681-692.

Giuseppe, C.M., Rita, G. and Giuseppe, P. (2008), "Stochastic optimum design of linear tuned mass damper for seismic protection of high towers", Struct. Eng. Mech., 29(6), 603-622.

Gong, X., Peng, C., Xuan, S., Xu, Y. and Xu, Z. (2012), "A pendulum-like tuned vibration absorber and its application to a multi-mode system”, J. Mech. Sci. Tech., 26(11), 3411-3422.

Hashem, H. and Hessamoddin, M.R. (2014), "Seismic control response of structures using an ATMD with fuzzy logic controller and PSO method”, Struct. Eng. Mech., 51(4), 547-564.

Haupt, R.L. and Haupt, S.E. (2004), Practical Genetic Algorithm, John Willey \& Sons, USA.

$\mathrm{Hu}, \mathrm{Z}$., Wang, M. and Zan, T. (2013), "Dynamic vibration absorber design to suppress boring chatter: absorber parameters identification based on modal correlation”, Adv. Mat. Res., 753-755, 1816-1820.

Nigdeli, S.M. and Bekdas, G. (2013), "Optimum tuned mass damper design for preventing brittle fracture of RC building”, Smart Struct. Syst., 12(2), 137-155.

Nigdeli, S.M. and Bekdas, G. (2014), “Optimum tuned mass damper approaches for adjacent structures", Earthq. Struct., 7(6), 1071-1091.

Seto, K., Iwasaki, Y., Shimoda, I., Oda, S. and Watanabe, T. (2011), "Vibration control for house structures beyond 3 story using adjustable pendulum-type controller under ground excitation like traffic vibrations or earthquakes", J. Syst. Des. Dyn., 5(5), 653-664.

Seto, K. and Ookuma, M., Yamashita, S. and Nagamatsu, A. (1987), "Method of estimating equivalent mass of multi-degree-of-freedom system", JSME Int. J., 30, 1638-1644.

Tributsch, A. and Adam, C. (2012), "Evaluation and analytical approximation of tuned mass damper performance in an earthquake environment", Smart Struct. Syts., 10(2), 155-179.

Wu, C.J., Chang, C.H. and Lin, Y.Y. (2009), "Optimal design for non uniform tuned liquid column damper in horizontal motion", J. Sound Vib., 326, 104-122

Xiang, P. and Nishitani, A. (2015), "Optimum design and application of non-traditional tuned mass damper toward seismic response control with experimental test verification”, Earthq. Eng. Struct. Dyn., 44(13), 2199-2220.

Yang, F., Sedaghati, R. and Esmaelzadeh, E. (2015), "Optimal design of distributed tuned mass dampers for passive vibration control of structures", Struct. Control Hlth. Monit., 22(2), 221-236.

Yu, J., Yamaura, H., Oishi, T. and Yu, Y. (2013), "Vibrations suppression control of image transfer belt system with flywheel or dynamic vibration absorber", J. Adv. Mech. Des. Syst. Man., 7, 52-64.

Zhul, F., Wang, J.T., Jin, F. and Altay, O. (2015), "Real-time hybrid simulation of single and multiple tuned column dampers for controlling seismic-induced response", 6th International Conference on Advances in Experimental Structural Engineering, Illinois, USA, August. 